\title{
Análisis de la Relación entre la Victimización y la Violencia Escolar: El Rol de la Reputación Antisocial
}

\section{Analysis of the Relationship Between Victimization and School Violence: The Role of Antisocial Reputation}

\author{
Estefanía Estévez ${ }^{1}$, Cándido J. Inglés ${ }^{1}$, Nicholas P. Emler ${ }^{2}$, María C. Martínez-Monteagudo ${ }^{1}$ \\ y María S. Torregrosa ${ }^{1}$ \\ ${ }^{1}$ Universidad Miguel Hernández de Elche, España \\ ${ }^{2}$ University of Surrey, UK
}

\begin{abstract}
Resumen. Estudios previos han identificado dos subgrupos de víctimas de violencia escolar. Un primer subgrupo lo componen la mayoría de víctimas que se caracterizan por su respuesta pasiva y sumisa ante los ataques, mientras que un menor porcentaje, aunque en mayor riesgo de desajuste psicosocial, combina la victimización con un comportamiento desafiante, hostil y agresivo. El presente estudio se centra en el segundo caso, con el objeto de analizar los posibles factores influyentes en el proceso de transición de la victimización a la implicación en conductas agresivas en el contexto escolar. La premisa de partida se fundamenta en la teoría propuesta originalmente por Emler, que sostiene que la percepción de desprotección de la víctima en la situación de intimidación, puede resultar en la búsqueda de una reputación antisocial que le ayude a auto-defenderse ante futuros ataques, una imagen social que se configura precisamente con la implicación en actos violentos. Para analizar estas relaciones, se utilizó una muestra de 1795 adolescentes de entre 11 y 18 años, escolarizados en nueve centros de educación secundaria obligatoria. Para el análisis de los datos, se calculó un modelo de ecuaciones estructurales que confirmó la hipótesis de partida.

Palabras clave: adolescencia, ajuste psicosocial, reputación social, victimización, violencia escolar.
\end{abstract}

\begin{abstract}
Previous studies have identified two subgroups of victims of school violence. A first subgroup is composed of the majority of victims who are characterized by their passive response and withdrawal in violent situations, while a lower percentage, although at more risk of psychosocial maladjustment, combines victimization with a defiant, hostile and aggressive behaviour. The present study is focused on the second case, with the purpose of analyzing the possible factors influencing the transition from passive victimization to involvement in aggressive behaviors within the school context. The starting premise is based on the theory originally proposed by Emler, which posits that the victim's perception of helplessness in situations of intimidation, may result in the searching of an antisocial reputation that helps victims defend themselves against future attacks; a social image that takes shape precisely through the involvement in violent acts. To test these relationships, 1795 adolescents between 11 and 18 years of age were recruited from nine secondary schools. To analyse the data, a structural equation model was calculated, which confirmed the starting hypothesis.

Keywords: adolescence, psychosocial adjustment, social reputation, school violence, victimization.
\end{abstract}

El rol de la reputación antisocial La violencia escolar constituye actualmente uno de los principales retos del sistema educativo y de las administraciones públicas en materia de educación, por tratarse de un comportamiento con consecuencias notablemente negativas en el ajuste psicosocial de los alumnos victimizados (Cook, Williams, Guerra, Kim y Sadek, 2010). Existe cierto consenso en definir la violencia escolar

Correspondencia: Dra. Estefanía Estévez López. Área de Psicología Evolutiva y de la Educación. Departamento de Psicología de la Salud. Universidad Miguel Hernández de Elche. Av. Universidad, s/n, 03202, Elche (Alicante). E-mail: eestevez@umh.es como aquella conducta intencionada mediante la cual se causa un daño a otra persona dentro del centro educativo o en cualquier actividad organizada por éste, pudiendo ser ejercida o padecida por cualquier miembro de la comunidad educativa (Guerra et al., 2011). Este tipo de conducta se ha analizado en la literatura científica atendiendo principalmente dos cuestiones fundamentales: su naturaleza y su función (Little, Henrich, Jones, and Hawley, 2003).

Little et al. (2003) distinguen, por un lado, 3 tipos de comportamiento violento en función de su naturaleza o forma en que se manifiesta: física, verbal y relacional. Según estos autores, la violencia física hace referencia a la acción que se realiza a través de 
un contacto directo entre agresor y víctima (ej. golpes y empujones); la violencia verbal se refiere a la acción que pretende causar daño a través de la palabra (ej. insultos y amenazas); y la violencia relacional se define como aquel acto que tiene la intención de dañar la red social de amistades de la víctima o su pertenencia a un grupo (ej. exclusión social o expandir falsos rumores sobre la víctima) (Estévez, Murgui, Musitu y Moreno, 2008a). La mayor parte de los trabajos en este ámbito se ha centrado en examinar el perfil de aquellos alumnos que presentan problemas de violencia física o verbal, mientras que el estudio de la violencia relacional ha quedado tradicionalmente relegado en la literatura científica, lo que contrasta con su importante prevalencia en las escuelas y su influencia en el ajuste psicosocial de los implicados (Crick y Grotpeter, 1996). En esta línea, estudios recientes apuntan que la violencia relacional es más sutil y menos visible que las formas física y verbal, aunque puede tener consecuencias tan negativas para el ajuste psicosocial del adolescente como las derivadas de la violencia manifiesta (Moreno, Estévez, Murgui y Musitu, 2009). De hecho, los escasos estudios previos que se han llevado a cabo en este ámbito han mostrado la existencia de una estrecha asociación entre la conducta violenta relacional y determinadas características psicosociales de los alumnos victimizados, como su reputación social en el grupo de iguales (Cillessen y Mayeux, 2004; Nelson, Robinson y Hart, 2005; Rose, Swenson y Waller, 2004), el sentimiento de soledad experimentado (Crick y Grotpeter, 1996) y otros problemas de ajuste emocional como la baja autoestima (Crick y Nelson, 2002; Villarreal-González, Sánchez-Sosa, Veiga y Del Moral, 2011).

Por otro lado, desde la perspectiva motivacional, Little et al. (2003) clasifican el comportamiento violento atendiendo a sus tres funciones principales: pura, reactiva e instrumental. La violencia pura es definida como aquella cuyo propósito es la agresión en sí misma; la violencia reactiva como el comportamiento que responde a una provocación o amenaza previa; y la violencia instrumental como aquel comportamiento premeditado cuyo objetivo es satisfacer un propósito o interés particular del agresor (Cosi, Vigil-Colet, y Canals, 2009).

El presente trabajo se adscribe a la propuesta de Little et al. (2003) que aglutina las seis dimensiones del comportamiento violento previamente descritas. En este sentido, es importante resaltar que son muy escasos los trabajos en materia de violencia escolar que consideran una definición tan amplia e integrante de esta expresión comportamental, por lo que entendemos que este aspecto constituye una de las principales contribuciones del presente estudio a la literatura científica en el ámbito de trabajo que nos ocupa. Y es que todos los tipos y motivaciones de la violencia han puesto de manifiesto su influencia perjudicial y grave impacto negativo en el ajuste psicosocial de la persona atacada (Juvonen, Nishina y Graham, 2000; Kumpulainen, Räsänen y Puura, 2001; Kupersmidt, Coie y Dodge, 1990). Así, diversos estudios han encontrado una estrecha asociación entre la victimización escolar y la baja autoestima (Austin and Joseph, 1996; Cava, Musitu, and Murgui, 2007; Guterman, Hahm and Cameron, 2002), especialmente en la dimensión social de este constructo (Andreou, 2000; Estévez, Martínez y Musitu, 2006; O’Moore y Kirkman, 2001); a este respecto, se ha observado una asociación entre las malas relaciones en el grupo de iguales y la aparición de abusos de poder e intimidación entre compañeros (Romera, Del Rey y Ortega, 2011).

Otros trabajos han documentado la relación existente entre sufrir acoso escolar y la baja satisfacción con la vida (Martin, Huebner y Valois, 2008; Martínez, Moreno, Amador y Orford, 2011; Povedano, Hendry, Ramos y Varela, 2011), así como la presencia de sintomatología depresiva (Herrero, Estévez y Musitu, 2006; Sweeting, Young, West, and Der, 2006; Kumpulainen et al., 2001). Un reciente enfoque apunta incluso que la victimización puede ejercer un impacto negativo en la capacidad empática de la víctima quien, lejos de comprender el comportamiento del agresor, intentará defenderse mostrando una conducta igualmente hostil (Emler, 2009). Este sería el caso específico del subgrupo de víctimas que ha venido a denominarse víctima agresiva, sobre el que profundizamos en mayor medida en el siguiente epígrafe.

\section{Subgrupos de víctimas de violencia escolar}

Son numerosos los estudios sobre violencia escolar que han concluido que las víctimas constituyen un grupo homogéneo en cuanto a sus atributos y características. Sin embargo, desde hace más de dos décadas encontramos trabajos en la literatura científica que señalan la existencia de, al menos, dos subtipos de víctimas de violencia escolar (Schwartz, 1999), que se diferencian fundamentalmente por su manera de reaccionar ante la situación de violencia (Estévez, Jiménez y Moreno, 2011). La mayoría de víctimas se caracteriza por mostrar sumisión y pasividad frente al agresor (Schwartz, Proctor y Chien 2001), pero existe una pequeña proporción de víctimas, identificada por vez primera por Olweus en 1978, que muestran a su vez un comportamiento hostil y agresivo. Las primeras han recibido el nombre de víctimas puras, víctimas sumisas o simplemente víctimas, mientras que a las segundas se les denomina en diversos trabajos como víctimas agresivas, víctimas provocadoras o bully-víctimas (Boulton y Smith, 1994; Estévez, Musitu y Murgui, 2008; Olweus, 2001; Schwartz et al., 2001).

Aunque los datos disponibles sobre la prevalencia 
de estos subgrupos de víctimas son ciertamente escasos, los pocos estudios disponibles apuntan que las víctimas sumisas representan una mayor proporción que las víctimas agresivas, quienes constituyen aproximadamente el 10-20\% del total (Olweus, 2001). Sin embargo, estas últimas parecen constituir un grupo en mayor riesgo de desarrollo de problemas de desajuste emocional y psicosocial (Estévez et al., 2008; Schwartz, 1999). En estudios comparativos entre ambos subtipos de víctimas se ha concluido que las víctimas agresivas, en comparación con las sumisas, presentan un perfil psicosocial más deteriorado: obtienen índices más bajos en medidas de autoestima (Estévez et al., 2006), niveles más elevados de depresión (Nansel et al., 2001; Schwartz, 1999) y presentan un mayor aislamiento social (Veenstra et al., 2005). Olweus (2007) señala que el perfil de la víctima agresiva combina los aspectos más negativos de las víctimas puras y los agresores, lo que supone, por tanto, no sólo un importante desajuste emocional, sino también un déficit notable en habilidades sociales tan esenciales para la interacción entre iguales como lo es la capacidad empática (Inglés, 2009).

\section{El presente estudio}

Este estudio se centra en el caso particular de las víctimas agresivas $\mathrm{y}$, específicamente, en el proceso explicativo vinculado al desarrollo de un comportamiento agresivo en una víctima de violencia escolar. Aunque podemos encontrar numerosos trabajos en la literatura científica que muestran la estrecha asociación existente entre la victimización escolar y el desarrollo de problemas internalizantes (ver Hawker y Boulton, 2000, para una revisión), pocos estudios han considerado la victimización como un factor de riesgo frente al desarrollo de problemas externalizantes como la violencia. Sí existe algún estudio longitudinal donde se concluye que la victimización física y verbal puede constituir un predictor del comportamiento agresivo y delincuente (p.ej. Hodges, Boivin, Vitaro y Bukoswki, 1999; Paul y Cillesen, 2003). Incluso más recientemente, Sullivan, Farrel y Kliewer (2006) encontraron resultados similares en un estudio transversal pero que incluía la violencia relacional. Sin embargo, ninguno de estos trabajos aporta una hipótesis explicativa de los mecanismos influyentes que derivan de un estado (ser víctima) al otro (mostrar una conducta de carácter agresivo).

Este trabajo parte del supuesto de que el vínculo victimización-violencia se explica, al menos en parte, por el desajuste psicosocial derivado del hecho de ser víctima, así como por otro factor tradicionalmente relegado en los estudios en este ámbito: la reputación social. La investigación previa sobre reputación y conducta delictiva llevada a cabo principalmente en Reino Unido y Australia por autores como Barry
(2006), Emler y Reicher (1995, 2005) y Carroll et al. (2001), revela interrelaciones entre estas variables bajo la siguiente argumentación avalada empíricamente en los trabajos mencionados: Cuando el adolescente es víctima de acoso o maltrato o se percibe en una situación de riesgo y amenaza ante los iguales y, además, no dispone del apoyo de una red social de protección adecuada (grupo de amigos, padres, profesores), puede recurrir a la búsqueda de una alternativa informal de protección frente a las situaciones de ataque. Así, el adolescente intentará configurarse una determinada reputación social fundamentada en una imagen de persona rebelde, anticonformista, y antisocial, una imagen que lleva implícita la idea de que la persona es fuerte, valiente y está dispuesta a vengarse de modo violento si fuera atacada de nuevo. Según Emler (2009) la estrategia más eficaz para configurar este tipo de reputación es precisamente la implicación en conductas violentas que ejemplifiquen lo que el adolescente quiere transmitir sobre su persona, bajo la asunción de que los agresores no pueden ser victimizados.

Los datos indican que la mayoría de niños victimizados no percibe disponer de una red social de apoyo y protección sino que, muy al contrario, se sienten desamparados ante la situación de maltrato o violencia y no suelen informar de estas vejaciones. En el trabajo desarrollado en nuestro contexto nacional por Serrano e Iborra (2005), se señala que tan sólo 3 de cada 10 víctimas de violencia escolar comunica a sus padres su problemática, y 4 de cada 10 a sus profesores. Este hecho acrecienta la probabilidad de que el caso no sea detectado por las figuras adultas más cercanas al alumno victimizado y que, por tanto, éste siga padeciendo los ataques de los iguales sin protección institucional, un aspecto que, a su vez, puede agravar las consecuencias negativas en el ajuste psicosocial de la persona.

El objetivo del presente estudio fue poner a prueba la argumentación de Barry (2006), Emler y Reicher $(1995,2005)$ y Carroll et al. (2001) en nuestro entorno educativo, considerando que sus supuestos pueden ser un adecuado punto de partida para el estudio de la relación entre la victimización y la violencia en estudiantes españoles de educación secundaria. Así, siguiendo a estos autores y teniendo en cuenta los hallazgos comentados en estudios previos, planteamos la hipótesis de que la asociación victimización-violencia se explica, al menos en parte, por: (1) las consecuencias negativas derivadas de la victimización sobre el ajuste psicosocial de la víctima a nivel de autoestima social, sintomatología depresiva, satisfacción vital y capacidad empática y, (2) el impacto que este desajuste psicosocial puede tener en la reputación social de la víctima y, en particular, en la decisión de ésta de optar por la alternativa de fraguarse una imagen antisocial entre sus iguales a través del comportamiento agresivo hacia los demás. 


\section{Método}

\section{Muestra}

En este estudio participaron 1795 adolescentes de ambos sexos (52\% varones), con edades comprendidas entre los 11 y 18 años $(M=14.2, D T=1.68)$ y escolarizados en 9 centros educativos (públicos y concertados) de la Comunidad Autónoma de Andalucía. Los participantes pertenecían a los cursos de $1^{\circ}, 2^{\circ}, 3^{\circ}$ y $4^{\circ}$ de la ESO y $1^{\circ}$ y $2^{\circ}$ de Bachillerato. La selección de los participantes se realizó mediante un muestreo estratificado por conglomerados (Santos, Muñoz, Juez y Cortiñas, 2003). Las unidades de muestreo fueron los centros educativos, públicos y concertados de entornos rurales y urbanos de colegios e institutos de la Comunidad Autónoma de Andalucía, para lo cual se utilizó el listado de centros educativos de esta comunidad. Los estratos se establecieron en función de la variable curso.

\section{Instrumentos}

Escala de Satisfacción con la Vida (Diener, Emmons, Larsen y Griffin, 1985; versión española de Atienza, Pons, Balaguer y García-Merita, 2000). Esta escala está compuesta por 5 ítems que proporcionan un índice general de satisfacción con la vida referido al bienestar subjetivo percibido (por ejemplo, "Mi vida es en la mayoría de los aspectos como me gustaría que fuera"). El rango de respuesta oscila entre 1-muy en desacuerdo- y 4 -muy de acuerdo-. Con relación a la validez de la escala, se han observado correlaciones negativas entre esta medida de satisfacción vital y otras de sentimiento de soledad, estrés percibido, sintomatología depresiva, y conducta violenta entre adolescentes (Buelga, Musitu y Murgui, 2009; Estévez et al., 2009) y asociaciones positivas con la percepción de un clima familiar comunicativo y cálido, y autoestima social (Martínez, Buelga y Cava, 2007). La fiabilidad constatada a través del alfa de Cronbach en el presente estudio fue de .74 .

Dimensión de Autoestima Social del Cuestionario AF5 (García y Musitu, 2009). La subescala de autoestima social de este instrumento consta de 6 ítems que informan de la percepción de auto-competencia en las relaciones sociales. Los ítems tienen una escala de respuesta de 1 -nunca- a 5 -siempre-. La dimensión social está integrada por dos aspectos: uno referido a la red social del sujeto y a su facilidad o dificultad para mantenerla o ampliarla (por ejemplo, "Consigo fácilmente amigos/as"), y otro concerniente a cualidades personales de importancia para las relaciones interpersonales (por ejemplo, "Soy un chico/a alegre"). Respecto de la validez del instrumento, puntuaciones elevadas en autoestima social han mostrado correlaciones negativas con medidas de estatus rechazado en el aula, sintomatología depresiva y comportamiento violento (Estévez, Herrero, Martínez y Musitu, 2006; Estévez, Martínez y Musitu, 2006). La consistencia interna de esta subescala medida a través del alfa de Cronbach en la presente muestra fue de .74.

Escala De Depresión del Centro De Estudios Epidemiológicos de Estados Unidos (CES-D; Radloff, 1977). La CES-D se compone de 22 ítems que evalúan de 1 -nunca o muy rara vez-a 4 -siempre o la mayoría de veces- la presencia de sintomatología depresiva. El CESD incluye varias dimensiones: ánimo deprimido, sentimientos de culpa e inutilidad, pérdida de apetito, desamparo y desesperación, problemas de sueño, y problemas de concentración, aunque la escala también proporciona un índice general de ánimo depresivo, que es el que se utiliza en la mayoría de las investigaciones, y también el referente del presente estudio. Este índice general no evalúa la depresión en sí misma, sino la sintomatología que habitualmente va asociada a ella (por ejemplo "Durante la última semana, me he sentido triste"). Esta escala ha sido ampliamente utilizada y validada en población adolescente y ha mostrado excelentes propiedades psicométricas (Meadows, Brown, y Elder, 2006; Wilcox, Field, Prodromidis y Scafidi, 1998). La sintomatología depresiva medida a través de este instrumento correlaciona en sentido positivo con escalas de estrés percibido, sentimiento de soledad, problemas de comunicación familiar, victimización y violencia escolar (Cava, Buelga, Musitu y Murgui, 2010; Estévez, Murgui, Musitu y Moreno, 2008b; Herrero, Estévez y Musitu, 2006). La fiabilidad del instrumento obtenida para esta muestra, según el alfa de Cronbach, fue de .81 .

Dimensión Self Público Ideal de la Escala de Reputación Social (Carroll, Hattie, Durkin y Houghton, 1999; traducción bidireccional inglés-español). Esta dimensión consta de 7 ítems que evalúan con un rango de respuesta que va de 1 -nunca- a 4 -siempre-, la percepción del adolescente de su reputación ideal antisocial entre el grupo de iguales (por ejemplo "Me gustaría que los demás pensasen que soy un matón", "Me gustaría que los demás pensasen que hago cosas en contra de la ley"). Con respecto a la validez de esta medida, se han observado correlaciones estadísticamente significativas en sentido positivo con delincuencia autoinformada, conducta violenta y consumo de sustancias (Buelga et al., 2009; Carroll, Green, Houghton y Wood, 2003) y en sentido negativo con índices de soledad y satisfacción vital (Buelga, Musitu, Murgui y Pons, 2008; Moreno, Estévez et al., 2009). El alfa de Cronbach obtenido en esta investigación fue de .80 .

Índice de Empatía para Niños y Adolescences (IECA; Bryant, 1982). El IECA es una adaptación para población infantil y adolescente de la escala para adultos de Mehrabian y Epstein (1972). Está adaptada al español por Mestre, Pérez-Delgado, Frías y Samper (1999) y consta de 22 ítems que aportan, en una esca- 
la de respuesta de 1 -nunca-a 4 -siempre-, un índice general del grado de empatía experimentado por el adolescente en distintas situaciones planteadas (por ejemplo "Me siento mal cuando veo que se hace daño a una chica"). Esta escala ha sido ampliamente utilizada y validada en muestras de adolescentes, mostrando propiedades psicométricas excelentes (Mestre, Samper, Frías y Tur, 2009; Miller y Eise nberg, 1986). Se han obtenido correlaciones positivas con otras medidas de empatía (Wied, Goudena y Matthys, 2005) y de altruismo (Mestre, Pérez, Samper y Martí, 1998), y correlaciones negativas con escalas de conducta antisocial (Sobral, Romero, Luengo y Marzoa, 2000) y conducta violenta (Estévez et al., 2008a; Wied et al., 2005). La escala presentó un coeficiente de fiabilidad, alfa de Cronbach, para la presente muestra de .77 .

Escala de Victimización.Este instrumento fue elaborado ex professo a partir de la Escala Multidimensional de Victimización de Mynard y Joseph (2000) y el Cuestionario de Experiencias Sociales de Crick y Grotpeter (1996). Se compone de 20 ítems que describen situaciones de victimización directa e indirecta entre los iguales (10 ítems corresponden a victimización directa y 10 a victimización indirecta), con una escala de respuesta tipo Likert de 1 -nunca- a 4 -muchas veces-. En un estudio previo (Cava, Musitu y Murgui, 2007) se realizó un análisis factorial con rotación oblimin que indicó una estructura de tres factores que explican el $62.18 \%$ de varianza: (1) Victimización Relacional, compuesto por 10 ítems (por ejemplo, "Algún compañero/a le ha dicho a los demás que no se relacionen conmigo"), explica el 49.26\% de varianza; (2) Victimización Física, compuesto por 5 ítems (por ejemplo, “Algún compañero/a me ha pegado o golpeado"), explica el $7.05 \%$ de varianza; y (3) Victimización Verbal, compuesto por 5 ítems (por ejemplo, "Algún compañero/a me ha insultado"), explica el $5.87 \%$. Esta estructura factorial del instrumento se ha replicado en la presente muestra mediante análisis factorial confirmatorio con el programa AMOS (software versión 6.0., Arbuckle 2005). El alfa de Cronbach obtenido para esta muestra fue $.87, .67$ y .89 respectivamente. Este cuestionario ha sido utilizado en estudios previos con población adolescente, mostrando una adecuada fiabilidad y correlaciones significativas con otros indicadores de desajuste psicosocial tales como estrés percibido, sintomatología depresiva y sentimiento de soledad (Cava et al., 2007; Cava et al., 2010; Estévez et al., 2009; Jiménez, Musitu, Ramos y Murgui, 2009).

Escala de Conducta Violenta en la Escuela (adaptada de Little, Henrich, Jones y Hawley, 2003). Este instrumento consta de 25 ítems que evalúan, con un rango de respuesta de 1 -nunca- a 4 -siempre-, 6 dimensiones de la conducta violenta. Los autores distinguen, por un lado, la Violencia Manifiesta o directa, en sus formas pura (por ejemplo, "Soy una persona que se pelea con los demás"), reactiva (por ejemplo, "Cuando alguien me hace daño o me hiere, le pego") e instrumental (por ejemplo, "Amenazo a otros para conseguir lo que quiero). Por otro lado, se evalúa la Violencia Relacional o indirecta, también en sus formas pura (por ejemplo, "Soy una persona que no deja a los demás que entren en su grupo de amigos/as"), reactiva (por ejemplo, "Cuando alguien me enfada, digo a mis amigos que no se relacionen con esa persona") e instrumental (por ejemplo, "Para conseguir lo que quiero, trato con indiferencia a los demás o dejo de hablar con ellos"). La estructura factorial original del instrumento se ha replicado en la presente muestra mediante análisis factorial confirmatorio con el programa AMOS (software version 6.0., Arbuckle 2005). El coeficiente de fiabilidad alfa de Cronbach obtenido fue de .88 para Violencia Manifiesta y .81 para Violencia Relacional. Con respecto a la validez del instrumento, las seis dimensiones han mostrado relaciones positivas con medidas de actitud negativa hacia la autoridad institucional, transgresión hacia las normas sociales, reputación antisocial, conflictos familiares, estrés percibido e insatisfacción con la vida (Buelga et al., 2008, 2009; Estévez et al., 2008; Jiménez, Moreno, Murgui y Musitu, 2008).

\section{Procedimiento}

Tras enviar una carta explicativa a los centros educativos participantes, donde se explicó el propósito y alcance del estudio, se contactó telefónicamente con la dirección de los centros y se concertó una entrevista con el director o directora en la que se explicó la investigación con detalle. Una vez acordada la participación voluntaria del centro, se hizo entrega de los consentimientos informados para los padres, que se enviaron mediante carta a través de la dirección de las escuelas. Tras la obtención de los permisos correspondientes, se realizó un seminario informativo con la plantilla de profesorado de cada centro donde se expusieron los principales objetivos y contenidos del estudio. La aplicación de los instrumentos se llevó a cabo por un grupo de investigadores expertos y entrenados. La batería de instrumentos se administró a los adolescentes en sus aulas habituales durante un período regular de clase. El orden de administración de los instrumentos se contrabalanceó en cada clase y centro educativo. Se informó a los adolescentes que la participación en la investigación era voluntaria y anónima. El estudio cumplió las normas éticas requeridas en la investigación con seres humanos, respetando los principios fundamentales incluidos en la Declaración Helsinki, en sus actualizaciones, y en las normativas vigentes: consentimiento informado y derecho a la información, protección de datos personales y garantías de confidencialidad, no discriminación, gratuidad y posibilidad de abandonar el estudio en cualquiera de sus fases. 


\section{Resultados}

\section{Análisis preliminares}

En primer lugar, se calcularon las correlaciones bilaterales de Pearson entre todas las variables objeto de estudio, que se corresponden con las dimensiones te. Los siete factores incluidos en el modelo fueron los siguientes: (1) Victimización, compuesto por tres variables observables o indicadores (victimización verbal, física y relacional), (2) Conducta violenta, compuesto por seis indicadores (violencia manifiesta pura, reactiva e instrumental, y violencia relacional pura, reactiva e instrumental), (3) Autoestima social,

Tabla 1. Correlaciones bilaterales de Pearson, medias y desviaciones típicas. Los valores por encima de la diagonal corresponden a los chicos y por debajo de la diagonal y en cursiva corresponden a las chicas

\begin{tabular}{|c|c|c|c|c|c|c|c|c|c|c|c|c|c|c|}
\hline Variables & 1 & 2 & 3 & 4 & 5 & 6 & 7 & 8 & 9 & 10 & 11 & 12 & 13 & 14 \\
\hline 1. Victimización verbal & 1 & $.67 * *$ & $.76 * *$ & $-.18 * *$ & $-.19 * *$ & -.02 & $.22 * *$ & $.09 *$ & $.25 * *$ & $.15^{* *}$ & $.23 * *$ & $.25^{* *}$ & $.21^{* *}$ & $.22 * *$ \\
\hline 2. Victimización física & $.63^{* *}$ & 1 & $.70 * *$ & $-.21 * *$ & $-.15^{* *}$ & -.04 & $.28 * *$ & $.18^{* *}$ & $.27 * *$ & $.15^{* *}$ & $.28 * *$ & $.27 * *$ & $.15^{* *}$ & $.23^{* *}$ \\
\hline 3. Victimización relacional & $.73 * *$ & $.53^{* *}$ & 1 & $-.20^{* *}$ & $-.18 * *$ & .04 & $.24 * *$ & $.11^{* *}$ & $.26 * *$ & $.13^{* *}$ & $.29 * *$ & $.32 * *$ & $.22 * *$ & $.30^{* *}$ \\
\hline 4. Autoestima social & -.02 & -.03 & .01 & 1 & $.28 * *$ & $.12 * *$ & $-.17 * *$ & $-.08 *$ & $-.14 * *$ & -.01 & $-13^{* *}$ & $-.21 * *$ & $-.11 * *$ & $-.15^{* *}$ \\
\hline 5. Satisfacción con la vida & $-.13 * *$ & $-.14^{* *}$ & $-.14 * *$ & $.20 * *$ & 1 & $.08 * *$ & $-.47 * *$ & $-.16^{* *}$ & $-.23 * *$ & $-.14 * *$ & $-.21^{* *}$ & $-.24 * *$ & $-.13^{* *}$ & $-.19 * *$ \\
\hline 6. Empatía & -.06 & $-.09 * *$ & -.04 & $.13 * *$ & $.09 * *$ & 1 & -.01 & $-.16^{* *}$ & $-.27 * *$ & $-.28 * *$ & $-.29 * *$ & $-.21 * *$ & $-.11^{* *}$ & $-.21^{* *}$ \\
\hline 7. Sintomatologia depresiva & $.14 * *$ & $.10^{* *}$ & $.18^{* *}$ & $-.10^{* *}$ & $-.49 * *$ & .06 & 1 & $.23^{* *}$ & $.25 * *$ & $.15^{* *}$ & $.24 * *$ & $.29 * *$ & $.16^{* *}$ & $.27 * *$ \\
\hline 8. Reputación no-conformista & .06 & $.06^{*}$ & .04 & $-.12 * *$ & $-.08^{*}$ & $-.10^{* *}$ & $.14^{* *}$ & 1 & $.40^{* *}$ & $.29 * *$ & $.30^{* *}$ & $.24 * *$ & $.12^{* *}$ & $.27 * *$ \\
\hline 9. Violencia manifiesta pura & $.21 * *$ & $.15^{* *}$ & $.14 * *$ & .03 & $-.23 * *$ & $-.18 * *$ & $.19^{* *}$ & $.30 * *$ & 1 & $.56 * *$ & $.67 * *$ & $.52 * *$ & $.32 * *$ & $.54 * *$ \\
\hline 10. Violencia manifiesta reactiva & $.13^{* *}$ & $.11 * *$ & $.09 * *$ & $.11 * *$ & $-.19 * *$ & $-.18^{* *}$ & $.14 * *$ & $.31 * *$ & $.59 * *$ & 1 & $.47^{* *}$ & $.33^{* *}$ & $.39 * *$ & $.35 * *$ \\
\hline 11. Violencia manifiesta instrumental & $.17^{* *}$ & $.16^{* *}$ & $.16^{* *}$ & .03 & $-.15^{* *}$ & $-.27 * *$ & $.14^{* *}$ & $.27 * *$ & $.63^{* *}$ & $.54^{* *}$ & 1 & $.66 * *$ & $.38^{* *}$ & $.72 * *$ \\
\hline 12. Violencia relacional pura & $.17 * *$ & $.13 * *$ & $.14 * *$ & -.01 & $-.15 * *$ & $-.21 * *$ & $.16^{* *}$ & $.19 * *$ & $.49^{* *}$ & $.37 * *$ & $.49 * *$ & 1 & $.45^{* *}$ & $.71 * *$ \\
\hline 13. Violencia relacional reactiva & $.13^{* *}$ & $.07 *$ & $.17 * *$ & -.04 & $-.10^{* *}$ & $-.07 *$ & $.12^{* *}$ & $.16^{* *}$ & $.33^{* *}$ & $.38 * *$ & $.37 * *$ & $.45 * *$ & 1 & $.46 * *$ \\
\hline 14. Violencia relacional instrumental & $.14^{* *}$ & $.11^{* *}$ & $.17^{* *}$ & .03 & $-.13 * *$ & $-.21^{* *}$ & $.14^{* *}$ & $.20 * *$ & $.45^{* *}$ & $.37 * *$ & $.64 * *$ & $.52 * *$ & $.43^{* *}$ & 1 \\
\hline Media chicas & 1.59 & 1.15 & 1.43 & 3.26 & 3.11 & 3.10 & 1.98 & 1.27 & 1.29 & 1.53 & 1.11 & 1.29 & 1.90 & 1.20 \\
\hline Desviación típica chicas & .54 & .27 & .43 & .47 & .58 & .28 & .58 & .29 & .31 & .51 & .23 & .32 & .50 & .32 \\
\hline Media chicos & 1.66 & 1.24 & 1.40 & 3.27 & 3.10 & 2.79 & 1.92 & 1.36 & 1.44 & 1.87 & 1.24 & 1.38 & 1.93 & 1.32 \\
\hline Desviación típica chicos & .57 & .37 & .42 & .47 & .57 & .30 & .56 & .44 & .42 & .63 & .41 & .43 & .52 & .44 \\
\hline
\end{tabular}

Nivel de significación: * $p<0.05, * * p<0.01 * *$

de los instrumentos de recogida de datos seleccionados. Estos análisis correlacionales se presentan en la Tabla 1, junto con los valores de las medias y desviaciones típicas para chicos y chicas por separado. Si bien la mayoría de correlaciones entre variables resultaron estadísticamente significativas, algunos valores indicaron ausencia de relación entre dimensiones en el análisis por géneros. Este fue el caso particular de la asociación entre la empatía y la victimización en los chicos, y de la autoestima social y la victimización en el caso de las chicas. Estos resultados se consideraron en los análisis subsiguientes.

\section{Un modelo estructural sobre vicitimización y conducta violenta}

El modelo de ecuaciones estructurales fue establecido con el programa EQS (Structural Equation Modelling Software) versión 6.1 (Bentler, 1995) con el fin de analizar la relación entre los factores que se presentan en la Tabla 2. En esta tabla se recogen los datos relativos a las cargas factoriales o saturaciones de cada variable observable en su correspondiente factor laten-
Tabla 2. Cargas factoriales y errores estándar robustos entre paréntesis

\begin{tabular}{lc}
\hline Variables & Cargas factoriales \\
\hline Victimización & \\
\hline Victimización verbal & $1^{\text {a }}$ \\
Victimización física & $.501(.031)$ \\
Victimización relacional & $.730(.032)$ \\
\hline Conducta violenta & \\
\hline Violencia manifiesta pura & $1^{\text {a }}$ \\
Violencia manifiesta reactiva & $1.189(.059)$ \\
Violencia manifiesta instrumental & $.989(.049)$ \\
Violencia relacional pura & $.912(.051)$ \\
Violencia relacional reactiva & $1.009(.060)$ \\
Violencia relacional instrumental & $.931(.051)$ \\
\hline Autoestima social & $1^{\text {a }}$ \\
Satisfacción con la vida & $1^{\text {a }}$ \\
Empatía & $1^{\text {a }}$ \\
Sintomatologia depresiva & $1^{\text {a }}$ \\
Reputación no-conformista & $1^{\text {a }}$ \\
\hline
\end{tabular}

Todos los coeficientes significativos a nivel $p<.001$

${ }^{\text {a} F i j a d o s ~ e n ~} 1.00$ durante la estimación. 
(4) Satisfacción con la vida, (5), Empatía, (6) Sintomatología depresiva, y (7) Reputación antisocial. Puesto que los cinco últimos factores se constituyeron de un solo indicador, presentaron una carga factorial de $1 \mathrm{y}$ un error asociado con valor 0 .

El coeficiente del estimador normalizado nos indicó la inexistencia de normalidad multivariante en los datos (Coeficiente de Mardia $=122.9418$ ), por lo que se consideraron los estimadores robustos del método de máxima verosimilitud para determinar la bondad de ajuste del modelo y la significación estadística de los coeficientes. Los índices obtenidos mostraron un ajuste aceptable a los datos: $\mathrm{S}-\mathrm{B} \chi^{2}=426.3839, \mathrm{gl}=64, p$ $<.001, \mathrm{CFI}=.92, \mathrm{IFI}=.93, \mathrm{NNFI}=.93$; $\mathrm{RMSEA}=$ .05. Para los índices CFI, IFI y NNFI se consideran aceptables valores superiores a .90 , y para el índice RMSEA valores iguales o inferiores a $.08(\mathrm{Hu}$ y Bentler, 1999). Este modelo explicó el 30\% de la varianza de la variable final, conducta violenta.

La figura 1 muestra la representación gráfica del modelo estructural final con los coeficientes estandarizados y su probabilidad asociada. En esta figura se pueden observar distintas relaciones de influencia tanto directa como indirecta en la conducta violenta en la escuela. Respecto de las relaciones directas, los resultados mostraron, en primer lugar, una estrecha asociación en sentido positivo entre la victimización escolar y el comportamiento violento $(\beta=.26, p<$ .001). Los resultados también mostraron relaciones directas de influencia de la satisfacción vital y la empatía en la conducta violenta en sentido negativo $(\beta=-.12, p<.001 ; \mathrm{y}(\beta=-.27, p<.001$, respectivamente), así como entre la reputación antisocial y la conducta violenta en sentido positivo $(\beta=.27, p<$ $.001)$.

El modelo también nos indicó la existencia de relaciones indirectas entre las variables objeto de estudio. La victimización en la escuela mostró una influencia negativa en la autoestima social $(\beta=-.14, p<.001)$, en la satisfacción con la vida $(\beta=-.20, p<.001)$, y en el grado de empatía $(\beta=-.06, p<.001)$, y una fuerte asociación positiva con la sintomatología depresiva $(\beta$ $=.23, p<.001)$. A su vez, el modelo señaló relaciones de influencia estadísticamente significativas de la autoestima social, la empatía y la sintomatología depresiva en la reputación ideal antisocial $(\beta=.05, p$ $<.001 ; \beta=-.19, p<.001 ; \mathrm{y} \beta=.21, p<.001$, respectivamente); y la reputación antisocial, tal y como hemos comentado, presentó una estrecha asociación directa con el comportamiento violento en la escuela $(\beta=.27, p<.001)$. La satisfacción con la vida resultó ser la única variable que no mostró una relación significativa con la reputación antisocial, sino que pareció incidir directa y negativamente en la conducta violenta $(\beta=-.12, p<.001)$.

Finalmente, se evalúo la invarianza factorial del modelo mediante un análisis multigrupo en función del género de los adolescentes, con el objeto de comprobar si las relaciones observadas entre las variables diferían entre chicos y chicas. Para llevar a cabo este análisis se estimaron dos modelos que se compararon a través del test de la diferencia en $\chi^{2}$. Los modelos fueron los siguientes: a) un primer modelo multigrupo que no impone ninguna igualdad entre las saturaciones y las correlaciones entre factores (modelo sin restringir o modelo de línea base); y b) un segundo modelo que

Figura 1. Modelo estructural final con coeficientes de relación entre factores

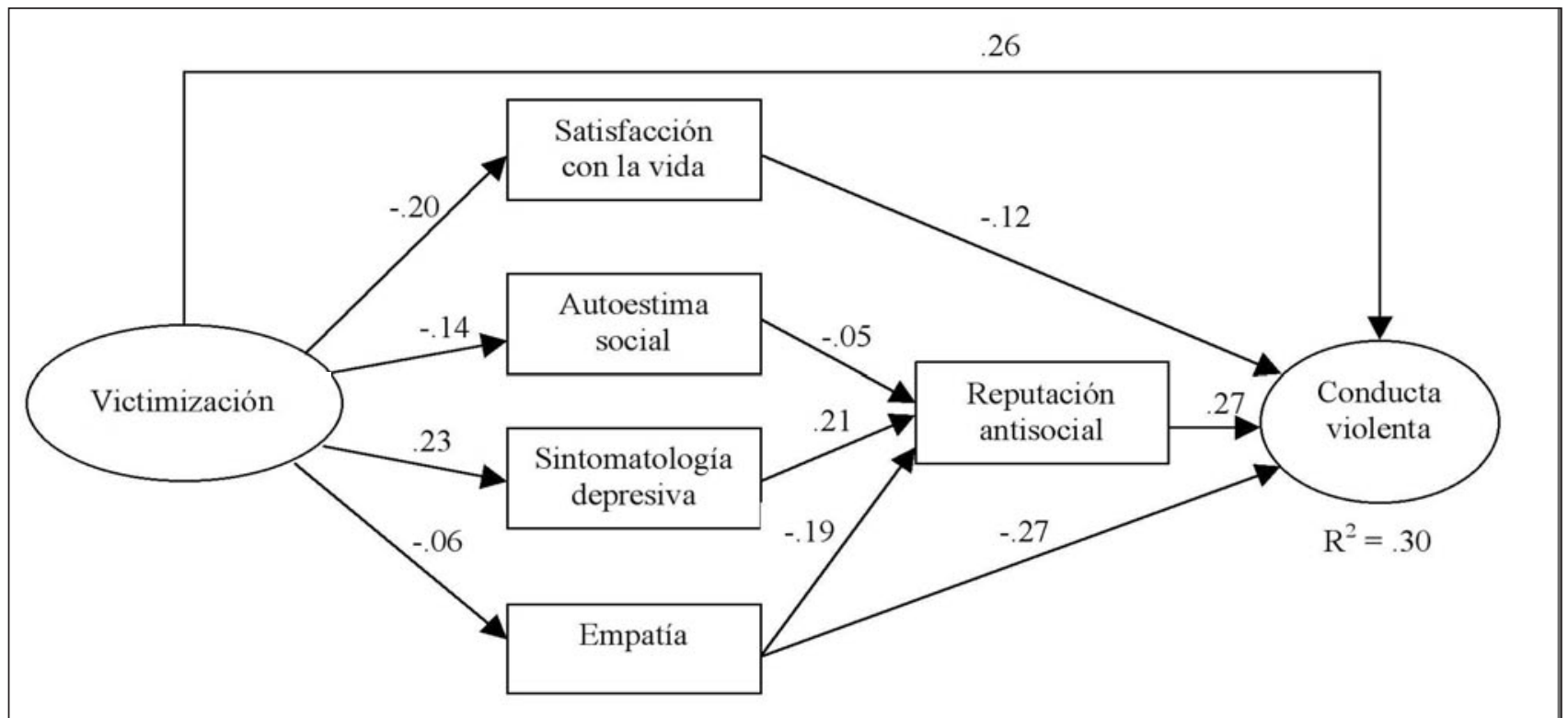

Las líneas continuas representan relaciones significativas entre variables.

La significación de las relaciones se ha determinado a partir del error estándar robusto.

Todos los coeficientes significativos a nivel $p<.001$ 
Tabla 3. Comparaciones multigrupos por género

\begin{tabular}{lrrrr}
\hline Modelo & S-B $\chi^{2}$ & gl & p & $\begin{array}{c}\text { CFI } \\
\text { robusto } \\
\text { robusto }\end{array}$ \\
\hline (a) Modelo sin restricciones & & 126 & .93 \\
(b) Modelo con restricciones & 412.0420 & 145 & $<.001$ & .05 \\
Modelo b - Modelo a & 554.7221 & 19 & .001 & .05 \\
(c) Modelo con restricciones liberadas & 142.6801 & $<.001$ & .92 \\
Modelo c - Modelo a & 469.8636 & 140 & $<.001$ & .05 \\
\hline
\end{tabular}

${ }^{1}$ Comparaciones basadas en el $\chi^{2}$ de Satorra-Bentler siguiendo el procedimiento descrito en Crawford y Henry (2003).

impone la igualdad entre todas las saturaciones y las correlaciones entre los factores en los grupos (modelo restringido). A continuación se examinó la diferencia entre los valores de $\chi^{2}$ y grados de libertad de ambos modelos para poder compararlos en términos de ajuste. En un último paso, se liberaron aquellas restricciones del modelo restringido que llevan a una pérdida de ajuste y se volvieron a examinar los parámetros individualmente para cada grupo. En la Tabla 3 se presenta un resumen de los índices de ajuste multigrupo obtenidos y del test de la diferencia en $\chi^{2}$.

La diferencia en el valor de $\chi^{2}$ entre el modelo restringido y sin restringir fue significativa $\left(\Delta \chi^{2}(19)=\right.$ $142.6801, p<.001$ ), lo que es indicativo de una invarianza métrica que señala la inexistencia de equivalencia en las relaciones observadas entre géneros. Con el fin de determinar qué elementos del modelo generaban estas diferencias, se analizaron los resultados del Test de los Multiplicadores de Lagrange (ML). Esta prueba reveló dos importantes diferencias significativas entre chicos y chicas: 1) la asociación entre la victimización y la autoestima social fue mucho mayor en los chicos $(\beta=-.24, p<.001)$ que en las chicas $(\beta=-.02, p<$ $.001)$; y 2) la relación entre la autoestima social y la reputación antisocial fue más elevada en el caso de las chicas $(\beta=.16, p<.001)$ en comparación con los chi$\cos (\beta=.02, p<.001)$. Una vez liberadas estas restricciones, el resto de relaciones resultaron estadísticamente equivalentes entre ambos géneros.

\section{Discusión}

En este estudio se ha considerado la victimización como un factor de riesgo frente al desarrollo de un comportamiento violento en la víctima de agresiones por parte de sus iguales en la escuela. En particular, el principal objetivo del trabajo fue poner a prueba la argumentación recientemente defendida por Emler (2009) y otros autores a partir de estudios con población delincuente juvenil acerca del vínculo victimización-violencia. Estos investigadores sostienen que la percepción de desprotección de la víctima en la situación de ataque o intimidación, puede resultar en la búsqueda de una reputación antisocial que le ayude a auto-defenderse ante futuros ataques, una imagen social que se configu- ra precisamente con la implicación en actos violentos que transmiten a los demás esa imagen de persona dura. Atendiendo además a los resultados constatados en números trabajos sobre la asociación entre la victimización y el desajuste psicológico y emocional en la adolescencia (por ejemplo, Cava et al., 2007; Kumpulainen et al, 2001; Sweeting et al., 2006), en el presente estudio se plantearon dos hipótesis de partida que se han visto confirmadas con los resultados obtenidos.

En el modelo estructural calculado observamos, en primer lugar, una relación directa, estrecha y en sentido positivo entre la victimización y la violencia escolar. Este resultado sugiere que el hecho de haber sido victimizado aumenta la probabilidad de desarrollar un comportamiento violento, o dicho con otras palabras, que la victimización en la escuela constituye un factor de riesgo para la conducta violenta en este contexto. La literatura científica relacionada en la que se analiza la victimización como variable de riesgo ante el desarrollo de problemas externalizantes, se limita a escasos estudios fuera de nuestro contexto nacional y, además, con población delincuente juvenil (Paul y Cillesen, 2003; Shaffer y Ruback, 2002; Smith y Ecob, 2007; Sullivan et al., 2006), un perfil diferente al utilizado en la presente investigación. Una contribución de este trabajo es, precisamente, la extrapolación de los hallazgos con jóvenes delincuentes al caso particular de adolescentes escolarizados víctimas de agresiones y, al tiempo, violentos con sus iguales.

Los resultados también han mostrado una relación indirecta entre la victimización y la violencia escolar a través de la influencia de otros factores considerados. Así, observamos, por un lado, una importante influencia negativa de la victimización en el ajuste psicosocial de la víctima y, en particular, en su satisfacción vital, autoestima social, sintomatología depresiva y capacidad empática, lo que confirma nuestra primera hipótesis. Estos resultados van en la línea de investigaciones previas en las que se ha mostrado la estrecha asociación existente entre el hecho de ser víctima de los iguales y distintos indicadores de desajuste emocional y psicológico (por ejemplo, Estévez et al., 2006; Herrero et al., 2006; Juvonen et al., 2000; Martin et al., 2008). A su vez, en el modelo calculado, alguno de estos indicadores como la satisfacción con la vida y, especialmente, la empatía, mostraron una fuerte asociación 
directa en sentido negativo con la conducta violenta. En esta línea, trabajos previos han documentado el vínculo entre el comportamiento violento hacia los demás y la presencia de déficits en la capacidad empática del agresor (Dykeman, Daehlin, Doyle y Flamer, 1996; Estévez et al., 2008a; Evans, Heriot y Friedman, 2002). El estudio de la satisfacción vital en la adolescencia es menos frecuente y los trabajos existentes analizan este constructo, principalmente, en el caso particular de las víctimas (Martínez-Antón, Buelga y Cava, 2007), aunque muy recientemente también encontramos trabajos donde se ha observado que los adolescentes violentos informan sentirse menos satisfechos con sus vidas (MacDonald, Piquero, Valois y Zullig, 2005, Moreno et al., 2009).

En la influencia indirecta observada de la victimización en la violencia, nuestros resultados también señalan el importante rol desempeñado por la reputación social de la víctima en la escuela. El modelo indicó que el desajuste emocional subsiguiente a la situación de victimización, operacionalizado en altas puntuaciones en sintomatología depresiva y bajas en autoestima social y empatía, puede devenir en la búsqueda de una reputación antisocial que derive, eventualmente, en el comportamiento violento, un proceso que confirma nuestra segunda hipótesis. Siguiendo la argumentación de Emler (2009) que tomamos como punto de partida en el presente estudio, la percepción de desamparo ante el ataque de los iguales es el principal motor que empuja al adolescente a fraguarse una reputación de persona intocable. Nuestros resultados van en la línea de las conclusiones de este autor, indicando que el malestar emocional que sufre la víctima, junto con la percepción de una ausencia de capital social donde apoyarse, en conjunto con la falta de empatía o la afectación negativa de ésta por la situación de victimización, conducen al deseo de fraguarse una imagen antisocial. Este deseo, denominado por algunos autores como reputación ideal antisocial, se ha asociado estrechamente a la conducta delictiva en la adolescencia (Carroll et al., 1999, 2003; Estévez y Emler, 2009). Nuestros resultados han mostrado que esta asociación también se extiende en casos de violencia escolar con adolescentes escolarizados y en ambos géneros.

Para finalizar, los autores reconocen algunas limitaciones que debieran ser consideradas para posteriores investigaciones. Una primera limitación es relativa a la muestra, que si bien recoge un número elevado de participantes $(N=1795)$, éstos pertenecen a una región geográfica concreta como es la Comunidad Autónoma de Andalucía, un aspecto que nos obliga a ser prudentes en la generalización de los resultados obtenidos a otros contextos. En segundo lugar, si bien los instrumentos seleccionados para la presente investigación han sido ampliamente utilizados y validados en población adolescente española, mostrando adecuadas propiedades psicométricas, en el caso particu- lar del presente estudio, la subescala de victimización física mostró un alpha de Cronbach de .67, un valor indicativo de una fiabilidad moderada, puesto que el criterio comúnmente aceptado en la comunidad científica para hablar de fiabilidad satisfactoria es, al menos, .70 (Field, 2005; Kline, 1999; Ponterotto y Ruckdeschel, 2007)

En tercer lugar, también es necesario señalar que la utilización de autoinformes para la recogida de datos, y en particular de medidas de comportamiento violento, puede afectar la validez de los resultados aumentando la probabilidad de obtener respuestas sesgadas. Los autoinformes, sin embargo, presentan ventajas muy notables que han generalizado su utilización en ciencias sociales, como permitir recoger información significativa en un período temporal corto, y facilitar la interpretación de los datos sin interpretaciones sujetas a inferencias. Aunque podría sugerirse la idoneidad de recopilar datos de otras fuentes como el profesorado para contrastar la documentación aportada en los autoinformes, también es cierto que algunos estudios sobre comportamientos de riesgo en la adolescencia donde se recogen datos de distintas fuentes (p.ej. padres e hijos), han demostrado la validez de las medidas de autoinforme (Flisher, Evans, Muller y Lombard, 2004; Ritakallio, Kaltiala- Heino, Kivivuori y Rimpelä, 2005). Autores como Kamphaus y Frick (2005) argumentan que es mucho más probable que los adolescentes sean precisos en los informes sobre su propio comportamiento, puesto que los padres pueden ser desconocedores, en muchos casos, de la frecuencia y magnitud del comportamiento antisocial del hijo.

Por último, la naturaleza transversal del estudio no nos permite determinar un orden causal en las relaciones encontradas. Así, aunque el modelo de ecuaciones estructurales calculado nos sugiere un orden en la secuencia de influencias, también es posible que las direcciones se establezcan en el sentido contrario o que muestren una asociación bidireccional, un aspecto que podría analizarse en mayor profundidad a partir de un diseño longitudinal. De hecho, existen trabajos con población juvenil delincuente que apuntan que la conducta antisocial aumenta el riesgo de ser victimizado (Deadman y MackDonald, 2004; Wittebrood y Niewbeerta, 1999).

A pesar de estas limitaciones, los autores consideran que el trabajo aquí presentado contribuye al conocimiento existente en la literatura científica sobre victimización y violencia escolar, destacando específicamente el rol desempeñado por la reputación social entre los iguales como mecanismo de conexión entre estas variables, y ampliando así las aportaciones de trabajos previos desarrollados fundamentalmente en Reino Unido y Australia con población adolescente delincuente. Así, los resultados obtenidos confirman, por un lado, la necesidad de incidir en el trabajo terapéutico con las víctimas a nivel emocional, y particularmente en su 
percepción de satisfacción vital y el desarrollo de síntomas de carácter depresivo. Por otro lado, sugieren también la recomendación de incluir en los programas sobre violencia escolar y, fundamentalmente de prevención para el desarrollo de un comportamiento hostil en los estudiantes victimizados, un factor a nivel psicosocial como es la reputación entre los iguales.

Emler y Reicher $(1995,2005)$ destacan el hecho de que la víctima recurre a la alternativa informal de protección mediante la configuración de una reputación antisocial debido a una causa fundamental: la percepción de ausencia de apoyo en la red social de la víctima. Este argumento ha sido confirmado en estudios previos recientes con escolares españoles (Cava, 2011; Estévez, Jiménez y Moreno, 2011), donde se pone de manifiesto la relevancia que tienen padres y profesores en el desarrollo de la percepción de protección versus desamparo en alumnos victimizados. La prevención de situaciones de gravedad en el contexto escolar debe tener también como protagonistas a los adultos, quienes se encuentran en la situación privilegiada de autoridad que puede defender y apoyar a la víctima y reeducar al agresor. A modo de conclusión, el indicador de la reputación social en la adolescencia, que ha sido tradicionalmente relegado en la investigación científica en nuestro contexto nacional en materia de violencia escolar, puede ser sin embargo muy significativo para la eficacia de los programas de intervención y prevención en el ámbito de la convivencia escolar.

\section{Referencias}

Andreou, E. (2000). Bully/Victim problems and their association with psychological constructs in 8-to 12-year old Greek schoolchildren. Aggressive Behavior, 26, 49-56.

Arbuckle, J. L. (2005). Amos 6.0 User's Guide. Chicago, IL: SPSS Inc.

Atienza, F. L., Pons, D., Balaguer, I., y García-Merita, M. (2000). Propiedades psicométricas de la escala de satisfacción con la vida en adolescentes. Psicothema, 12, 314320.

Austin, S. y Joseph, S. (1996). Assessment of bully/victim problems in 8 to 11 years old. British Journal of Educational Psychology, 66, 447-456.

Barry, M. (2006). Youth offending in transition: The search for social recognition. London: Routledge.

Boulton, M. J. y Smith, P.K. (1994). Bully/victim problems in middle-school children: Stability, self-perceived competence, peer perceptions, and peer acceptance. British Journal of Developmental Psychology, 12, 315-329.

Bryant, B. K. (1982). An Index of Empathy for Children and Adolescents. Child Development, 53 413-425.

Buelga, S., Musitu, G. y Murgui, S. (2009). Relaciones entre la reputación social y la agresión relacional en la adolescencia. International Journal of Clinical and Health Psychology, 9 (1), 127-141.

Buelga, S., Musitu, G., Murgui, S. y Pons, J. (2008).
Reputation, loneliness, satisfaction with life and aggressive behavior in adolescence. The Spanish Journal of Psychology, 11 (1), 192-200.

Carroll, A., Green, S., Houghton, S. y Wood, R. (2003). Reputation enhancement and involvement in delinquency among high school students. International Journal of Disability, Development and Education, 50, 253-273.

Carroll, A., Hattie, J., Durkin, K. y Houghton, S. (1999). Adolescent reputation enhancement: differentiating delinquent, nondelinquent, and at-risk youths. Journal of Child Psychology and Psychiatry, 40, 593-606.

Carroll, A., Houghton, S., Hattie, J. y Durkin, K. (2001). Reputation enhancing goals: integrating reputation enhancement and goal setting theory as an explanation of delinquent involvement. En F. H. Columbus (Ed.), Advances in psychology research (pp. 101-129). New York: Nova Science Publishers.

Cava, M. J. (2011). Familia, profesorado e iguales: Claves para el apoyo a las víctimas de acoso escolar. Psychosocial Intervention, 20, 183-192.

Cava, M. J., Buelga, S., Musitu, G. y Murgui, S. (2010). Violencia escolar entre adolescentes y sus implicaciones en el ajuste psicosocial: un estudio longitudinal. Revista de Psicodidáctica, 15 (1), 21-34.

Cava, M. J., Musitu, G. y Murgui, S. (2007). Individual and social risk factors related to overt victimization in a sample of Spanish adolescents. Psychological Reports, 101, 275-290.

Cillessen, A. H. N., y Mayeux, L. (2004). From censure to reinforcement: Developmental changes in the association between aggression and social status. Child Development, 75, 147-163.

Cook, C. R., Williams, K. R., Guerra, N. G., Kim, T. E. y Sadek, S. (2010). Predictors of bullying and victimization in childhood and adolescence: a meta-analytic investigation. School Psychology Quarterly, 25, 65-83.

Cosi, S., Vigil-Colet, A., y Canals, J. (2009). Desarrollo del cuestionario de agresividad proactiva/reactiva para profesores: estructura factorial y propiedades psicométricas. Psicothema, 21, 159-164.

Crawford, J. R. y Henry, J. D. (2003) The Depression Anxiety Stress Scales: Normative data and latent structure in a large non-clinical sample. British Journal of Clinical Psychology, 42, 111-131.

Crick, N. R., y Grotpeter, J. K. (1996). Children's treatment by peers: Victims of relational and overt aggression. Development and Psychopathology, 8, 367-380.

Crick, N. R., y Nelson, D. A. (2002). Relational and physical victimization within friendships: Nobody told me there'd be friends like these. Journal of Abnormal Child Psychology, 30, 599-607.

Deadman, D. y MacDonald, Z. (2004). Offenders as victims of crime?: An investigation into the relationship between criminal behaviour and victimization. Journal of the Royal Statistical Society, 167, 53-67.

Diener, E., Emmons, R., Larsen, R. J., y Griffin, S. (1985). The Satisfaction With Life Scale. Journal of Personalitiy Assessment, 49, 71-75. 
Dykeman, C., Daehlin, W., Doyle, S. y Flamer, H. S. (1996). Psychological predictors of school-based violence: Implications for school counselors. School Counselor, 44, 35-47.

Emler, N. (2009). Delinquents as a minority group: Accidental tourists in forbidden territory or voluntary émigrés? En F Butera y J. Levine (Eds.). Coping with minority status: Responses to exclusion and inclusion (pp. 127-154). US: Cambridge University Press.

Emler, N. y Reicher, S. (1995). Adolescence and delinquency. Oxford, UK: Blackwell Publishers Ltd.

Emler, N. y Reicher, S. (2005). Delinquency: Cause or consequence of social exclusion? En D. Abrams, M. A. Hogg y J. M. Marques (Eds.), The Social Psychology of Inclusion and Exclusion (pp. 211-241). New York: Psychology Press.

Estévez, E. y Emler, N. (2009). Individual differences in attitude to school and social reputation among peers: Implications for behavioural adjustment in educational settings. En J. E. Larson (Ed.), Educational Psycho-logy: Cognition and Learning, Individual Differences and Motivation (pp. 342-375). New York: Nova Science Publishers.

Estévez, E., Jiménez, T. y Moreno, D. (2011). Cuando las víctimas de violencia escolar se convierten en agresores: “¿Quién va a defenderme?”. European Journal of Education and Psychology, 3, 177-186.

Estévez, E., Herrero, J., Martínez, B. y Musitu, G. (2006). Aggressive and non-aggressive rejected students: an analysis of their differences. Psychology in the Schools, 43, 387-400.

Estévez, E., Martínez, B. y Musitu, G. (2006). La autoestima en adolescentes agresores y víctimas en la escuela: La perspectiva multidimensional. Intervención Psicosocial, 12, 32-41.

Estévez, E., Murgui, S. y Musitu, G. (2008). Psychosocial adjustment in aggressors, pure victims and aggressive victims at school. European Journal of Education and Psychology, 1 (2), 29-39.

Estévez, E., Murgui, S. y Musitu, G. (2009). Psychosocial adjustment in bullies and victims of school violence. European Journal of Psychology of Education, XXIV (4), 473-483.

Estévez, E., Murgui, S., Musitu, G. y Moreno, D. (2008a). Adolescent aggression: effects of gender and family and school environments. Journal of Adolescence, 31, 433450.

Estévez, E., Murgui, S., Musitu, G. y Moreno, D. (2008b) Clima familiar, clima escolar y satisfacción con la vida en adolescentes. Revista Mexicana de Psicología, 25 (1), 119-128.

Evans, M., Heriot, S. A. y Friedman, A. G. (2002). A behavioural pattern of irritability, hostility and inhibited empathy in children. Clinical Child Psychology and Psychiatry, 7, 211-224.

Field, A. (2005). Discovering statistics using SPSS. London: Sage Publications.

Flisher, A. J., Evans, J., Muller, M. y Lombard, C. (2004). Brief report: Test-retest reliability of self-reported adolescent risk behaviour. Journal of Adolescence, 27 (2), 207-212.

García, F. y Musitu, G. (2009). AF5: Autoconcepto Forma 5. Madrid: Tea.

Guerra, C., Álvarez-García, D., Dobarro, A., Núñez, J. C., Castro, L. y Vargas, J. (2011). Violencia escolar en estudiantes de Educación Secundaria de Valparaíso (Chile): Comparación con una muestra española. Revista Iberoamericana de Psicología y Salud, 2, 75-98.

Guterman, N. B., Hahm, H. C. y Cameron, M. (2002). Adolescent victimization and subsequent use of mental health counseling services. Journal of adolescent Health, 30, 336-345.

Hawker, D. S. J. y Boulton, M. J. (2000). Twenty years' research on peer victimization and psychosocial maladjustment: A meta-analytic review of cross-sectional studies. Journal of Child Psychology and Psychiatry, 41, 441455.

Herrero, J., Estévez, E. y Musitu, G. (2006). The relationships of adolescent school-related deviant behaviour and victimization with psychological distress: Testing a general model of the mediational role of parents and teachers across groups of gender and age. Journal of Adolescence, 29, 671-690.

Hodges, E., Boivin, M., Vitaro, F. y Bukowski, W. M. (1999). The power of friendship: Protection against an escalating cycle of peer victimization. Developmental Psychology, 35, 94-101.

Hu, L. y Bentler, P. M. (1999). Cut-off criteria for fit indices in covariance structure analysis: Conventional criteria versus new alternatives. Structural Equation Modeling, 6, $1-55$.

Inglés, C. J. (2009). Programa PEHIA : Enseñanza de Habilidades Interpersonales para Adolescentes. $3^{\mathrm{a}} \mathrm{ed}$. Madrid: Pirámide.

Jiménez, T. I., Moreno, D., Murgui, S. y Musitu, G. (2008). Factores psicosociales relacionados con el estatus social del alumno en el aula: El rol de la reputación social, la amistad, la conducta violenta y la relación con el profesor. International Journal of Psychology and Psychologica Therapy, 8, 227-236.

Jiménez, T. I., Musitu, G., Ramos, M. J. y Murgui, S. (2009). Community involvement and victimization at school: an analysis through family, personal and social adjustment. The Journal of Community Psychology, 37 (8), 959-974.

Juvonen, J., Nishina, A. y Graham, S. (2000). Peer harassment, psychological adjustment, and school functioning in early adolescence. Journal of Educational Psychology, 92, 349-359.

Kamphaus, R. W. y Frick, P. J. (2005). Clinical assessment of child and adolescent personality and behavior (2nd ed.). New York: Springer.

Kline, P. (1999). The handbook of psychological testing. London: Routledge.

Kumpulainen, K., Räsänen, E. y Puura, K. (2001). Psychiatric disorders and the use of mental health services among children involved in bullying. Aggressive Behavior, 27, 102-110. 
Kupersmidt, J. B., Coie, J. D. y Dodge, K. A. (1990). Predicting disorder from peer social problems. En S. R. Asher y J. D. Coie (Eds.), Peer rejection in childhood. New York: Cambridge University Press.

Little, T. D., Henrich, C. C., Jones, S. M. y Hawley, P. H. (2003). Disentangling the "whys" from the "whats" of aggressive behaviour. International Journal of Behavioral Development, 27, 122-133.

MacDonald, J., Piquero, A.,Valois, R. y Zullig, K. (2005). The relationship between life satisfaction, Risk-Taking Behaviors and Youth Violence. Journal of Interpersonal Violence, 20, 1495-1518.

Martin, K., Huebner, E. S. y Valois, R. F. (2008). Does life satisfaction predict victimization experiences in adolescence? Psychology in the Schools, 45, 705-714.

Martínez, M., Buelga, S. y Cava, M. J. (2007). La satisfacción con la vida en la adolescencia y su relación con la autoestima y el ajuste escolar. Anuario de Psicología, 38, 293-303.

Martínez, B., Moreno, D., Amador, L. V. y Orford, J. (2011). School victimization among adolescents. An analysis from an ecological perspective. Psychosocial Intervention, 20, 149-160.

Martínez-Antón, M., Buelga, S. y Cava, M. J. (2007). La satisfacción con la vida en la adolescencia y su relación con la autoestima y el ajuste escolar. Anuario de Psicología, 38(2), 293-303.

Meadows, S. O., Brown, J. S. y Elder, G. H. (2006). Depressive symptoms, stress, and support: gendered trajectories from adolescence to young adulthood. Journal of Youth and Adolescence, 35, 93-103.

Mehrabian, A. y Epstein, N. A. (1972). A measure of emotional empathy. Journal of Personality, 40 (4), 525-543.

Mestre, V., Pérez-Delgado, E., Frías, D. y Samper, P. (1999). Instrumentos de evaluación de la empatía. En E. PérezDelgado y V. Mestre, Psicología moral y crecimiento personal (pp. 181-190). Barcelona: Ariel.

Mestre, V., Pérez, E., Samper, P y Martí, M. (1998). Diferencias de género en la empatía y su relación con el pensamiento moral y el altruismo. Iber Psicología, 3 (1), $1-15$.

Mestre, M. V., Samper, P., Frías, M. D. y Tur, A. M. (2009). Are women more empathetic than men? A longitudinal study in adolescence. The Spanish Jouurnal of Psychology, 12, 76-83.

Miller, P. A. y Eisenberg, N. (1986). The relation of empathy to aggressive and externalizing/antisocial behavior. Psychological Bulletin, 103 (3), 324-344.

Moreno, D., Estévez, E., Murgui, S. y Musitu, G. (2009). Reputación social y violencia relacional en adolescentes: el rol de la soledad, la autoestima y la satisfacción vital. Psicothema, 21, 537-542.

Mynard, H. y Joseph, S. (2000). Development of the Multidimensional Peer Victimization Scale. Aggressive Behavior, 26, 169-178.

Nansel, T. R., Overpeck, M., Pilla, R. S., Ruan, W. J., Simons-Morton, B. y Scheidt, P. (2001). Bullying behaviors among US youth: Prevalence and association with psychosocial adjustment. Journal of the American Medical Association, 285, 2094-2100.

Nelson, D. A., Robinson, C. C., y Hart, C. H. (2005). Relational and physical aggression of preschool-age children: Peer status linkages across informants. Early Education and Development, 16, 115-139.

O’Moore, M. y Kirkham, C. (2001). Self-esteem and its relationship to bullying behavior. Aggressive behavior, 27, 269-283.

Olweus, D. (2001). Peer harassment: A critical analysis and some important issues. En J. Juvonen y S. Graham (Eds), Peer harassment in school: The plight of the vulnerable and victimized (pp. 3-20). New York: Guilford Press.

Olweus, D. (2007). Acoso escolar: hechos y medidas de intervención. Bergen: Publicaciones del Research Centre for Health Promotion.

Paul, J. J. y Cillessen, A. H. N. (2003). Dynamics of peer victimization in early adolescence: Results from a fouryear longitudinal study. Journal of Applied School Psychology, 19, 25-43.

Ponterotto, J. G. y Ruckdeschel, D. E. (2007). An overview of coefficient alpha and a reliability matrix for estimating adequacy of internal consistency coefficients with psychological research measures. Peceptual and Motor Skills, 105, 997-1014.

Povedano, A., Hendry, L. B., Ramos, M. J. y Varela, R. (2011). Victimización escolar: Clima familiar, autoestima y satisfacción con la vida desde una perspectiva de género. Psychosocial Intervention 20, 5-12.

Radloff, L. S. (1977). The CES-D scale: A self-report depression scale for research in the general population. Applied Psychological Measurement, 1, 385-401.

Ritakallio, M., Kaltiala-Heino, R., Kivivuori, J. y Rimpelä, M. (2005). Brief report: Delinquent behaviour and depression in middle adolescence: a Finnish community sample. Journal of Adolescence, 28 (1), 155-159.

Romera, E. M., Del Rey, R. y Ortega, R. (2011). Factores asociados a la implicación en bullying: Un estudio en Nicaragua. Psychosocial Intervention, 20, 161-170.

Rose, A. L., Swenson, L. P., y Waller, E. M. (2004). Overt and relational aggression and perceived popularity: Developmental differences in concurrent and prospective relations. Developmental Psychology, 40, 378-387.

Santos, J., Muñoz, A, Juez, P. y Cortiñas, P. (2003). Diseño de Encuestas para estudios de mercado. Técnicas de muestreo y análisis multivariante. Madrid: Editorial Ramón Areces.

Schwartz, D. (1999). Subtypes of victims and aggressors in children's peer groups. Journal of Abnormal Child Psychology 28, 181-192.

Schwartz, D., Proctor, L. J. y Chien, D. H. (2001). The aggressive victim of bullying: Emotional and behavioral dysregulation as a pathway to victimization by peers. En J. Juvonen y S. Graham (Eds), Peer harassment in school: The plight of the vulnerable and victimized (pp. 147-174). New York: Guilford Press.

Serrano, A. y Iborra, I. (2005). Violencia entre compañeros en la escuela. Valencia: Publicaciones del Centro Reina Sofía para el Estudio de la Violencia. 
Shaffer, J. N. y Ruback, R. B. (2002). Violent victimization as a rick factor for violent offending among juveniles. OJJDP Juvenile Justice Bulleting. Washington, D.C.: U.S. Department of Justice, Office of Juvenile Justice and Delinquency Prevention.

Smith, D. J. y Ecob, R. (2007). An investigation into causal links between victimization and offending in adolescents. The British Journal of Sociology, 58, 633-659.

Sobral, J., Romero, E., Luengo, A. y Marzoa, J. (2000). Personalidad y conducta antisocial: amplificadores individuales de los efectos contextuales. Psicothema, 4, 661670.

Sullivan, T., Farrell, A. D., y Kliewer, W. (2006). Peer victimization in early adolescence: Association between physical and relational victimization and drug use, aggression, and delinquent behaviors among urban middle school students. Development and Psychopathology, 18, 119-137.

Sweeting, H., Young, R., West, P. y Der, G. (2006). Peer victimization and depression in early-mid adolescence: A longitudinal study. British Journal of Educational Psychology, 76, 577-594.

Veenstra, R. Lindenberg, S., Oldehinkel, A. J., De Winter, A. F., Verhulst, F. C. y Ormel, J. (2005). Bullying and victimization in elementary schools: a comparison of bullies, victims, bully/victims, and uninvolved preadolescents. Developmental Psychology, 41, 672-682.

Villarreal-González, M. E., Sánchez-Sosa, J. C., Veiga, F. H. y Del Moral, G. (2011). Contextos de desarrollo, malestar psicológico, autoestima social y violencia escolar desde una perspectiva de género en adolescentes Mexicanos. Psychosocial Intervention, 20, 171-181.

Wied, M., Goudena, P. P. y Matthys, W. (2005). Empathy in boys with disruptive behavior disorders. Journal of Child Psychology and Psychiatry, 46 (8), 867-880.

Wilcox, H., Field. T., Prodromidis. M. y Scafidi. F. (1998). Correlations between BDI and CES-D in a sample of adolescent mothers. Adolescence, 33, 565-574.

Wittebrood, K. y Niewbeerta, P. (1999). Wages of sin?: the link between offending, lifestyle and violent victimization. European Journal of Criminal Policy and Research, 7, 63-80.

\section{Nota de los autores}

Este estudio ha sido elaborado en el marco del proyecto de investigación PSI2008-01535/PSIC: "Violencia escolar, victimización y reputación social en la adolescencia" subvencionado por el Ministerio de Ciencia e Innovación de España.

Manuscrito recibido: 02/02/2011 Revisión recibida: 23/09/2011 Manuscrito aceptado: 04/10/2011 